\title{
Análisis comparativo de las propiedades mecánicas determinadas con técnicas de nanoindentación ${ }^{(\bullet)}$
}

\author{
M.A. Garrido-Maneiro*, A.J. López-Galisteo* y J. Rodríguez
}

\begin{abstract}
Resumen En este trabajo se analizan las posibilidades de la nanoindentación para caracterizar el comportamiento mecánico de un material, comparando sus resultados con los que proporciona el ensayo de tracción convencional. Dada la imposibilidad de fabricar probetas de tracción a partir de recubrimientos, se han empleado como materiales de referencia dos aleaciones de aluminio con valores diferentes de módulo elástico, límite elástico e índice de endurecimiento. Posteriormente, la técnica se ha empleado en la caracterización de materiales base níquel depositados por proyección térmica.
\end{abstract}

Palabras clave Nanoindentación. Recubrimientos de níquel.

\section{Mechanical properties determined by nanoindentation tests}

\begin{abstract}
Mechanical properties obtained through nanoindentation tests have been compared to those provided by the traditional tensile tests. Due to the small dimensions of the coatings, two bulk aluminium alloys with different elastic constants, yield strength and hardening index were tested. The nanoindentation technique was also applied to characterize thermal sprayed nitrogen $(\mathrm{Ni})$ coatings.
\end{abstract}

Keywords Nanoindentation. Thermal sprayed nickel coatings.

\section{INTRODUCCIÓN}

El empleo de recubrimientos de todo tipo ha experimentado un considerable aumento, convirtiéndose en una práctica habitual en nuestros días en numerosas aplicaciones industriales. Los recubrimientos de aleaciones de base níquel son especialmente indicados para aquellos casos donde la resistencia a la corrosión y el desgaste son parámetros críticos de diseño.

En procesos donde la degradación mecánica es considerable suele ser necesario acudir a técnicas de simulación, como el método de los elementos finitos, para analizar la evolución del recubrimiento. Estas técnicas necesitan como entrada la ecuación constitutiva del material para poder predecir su evolución en función de la solicitación exterior. Dadas las dimensiones habituales de un recubrimiento, no suele existir la posibilidad de fabricar probetas convencionales de tracción, las cuales, ensayadas en una máquina universal de ensayos, proporcionarían la curva tensión-deformación del material depositado.

Las nuevas técnicas de nanoindentación constituyen un avance en la caracterización mecánica de recubrimientos. Básicamente, consisten en un ensayo de penetración con registro simultáneo de carga y desplazamiento. Las pequeñas cargas y la gran resolución en la medida del desplazamiento permiten ensayar el recubrimiento sin que el substrato influya en los resultados obtenidos. Desde los primeros trabajos publicados sobre la técnica de nanoindentación ${ }^{[1-3]}$ se conoce el procedimiento para determinar el módulo de elasticidad y la dureza y, en publicaciones más recientes ${ }^{\left[4 \text { y }^{5]}\right.}$, han aparecido intentos de determinar, a partir de los registros originales, los parámetros principales de la curva tensión/deformación: límite elástico e índice de endurecimiento del material. Aunque se ha avanzado considerablemente en esta dirección en los últimos años, lo cierto es que no existe un método generalmente válido para la determinación de

(•) Trabajo recibido el día 20 de junio de 2003 y aceptado en su forma final el día 16 de abril de 2004.

(*) Escuela Superior de Ciencias Experimentales y Tecnología, Universidad Rey Juan Carlos. C/ Tulipán s/n 28933 Móstoles, Madrid. 
la curva tensión-deformación completa a partir de los registros carga-desplazamiento obtenidos en ensayos de nanoindentación. Si esto fuera posible, la aplicación a recubrimientos no sería la única ventaja. Más trascendente aún, sería la posibilidad de disponer de un ensayo no destructivo de caracterización mecánica del material.

En este trabajo se analizan críticamente las posibilidades de la nanoindentación comparando directamente los resultados que proporciona con los correspondientes a ensayos de tracción de materiales monolíticos conocidos. Con posterioridad, se utilizará la técnica en recubrimientos $\mathrm{NiCrBSi}$, depositados por proyección térmica ${ }^{[6]}$.

\section{EXPERIMENTACIÓN}

\subsection{Aleaciones de aluminio}

La primera parte del trabajo experimental se centró en la caracterización de materiales masivos para comparar los datos proporcionados por los ensayos de nanoindentación con los resultados obtenidos en ensayos de tracción convencionales. Como materiales de referencia para el estudio, se trabajó con dos aleaciones de aluminio, Al 2011 y $\mathrm{Al} 8090$, de las que se han realizado ensayos de tracción a temperatura ambiente y se han determinado módulo de elasticidad, E, límite elástico, $\sigma_{\mathrm{y}}$, e índice de endurecimiento, $\mathrm{n}$. Las curvas tensión deformación y los valores más característicos obtenidos se recogen en la figura 1 .

A continuación se realizaron ensayos de nanoindentación de ambas aleaciones de aluminio.

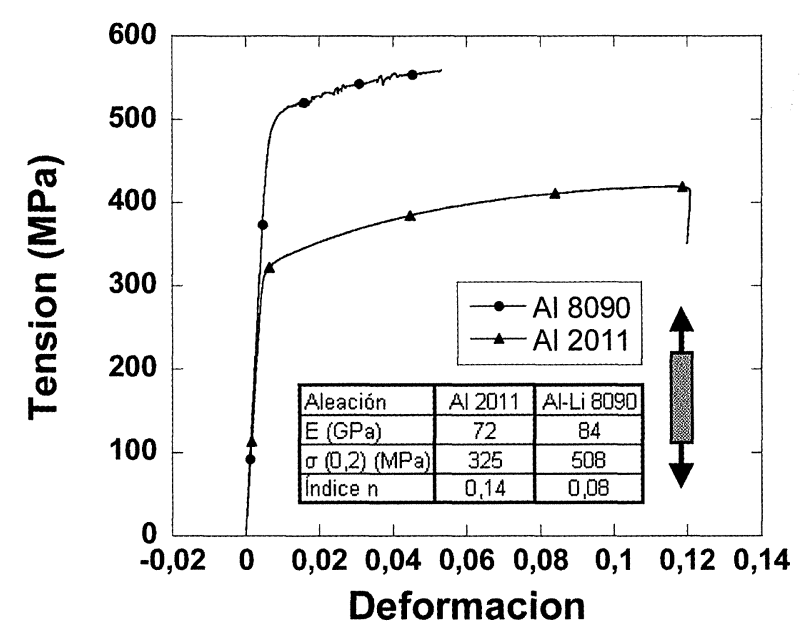

Figura 1. Curvas tensión-deformación de ensayos de tracción para las aleaciones de aluminio.

Figure 1. Stress strain-curves from tensile tests for aluminium alloys.
El nanoindentador empleado (NANOINDENTER XP de MTS) utiliza una punta Berkovich y proporciona curvas carga-desplazamiento (P-h) como las incluidas en la figura 2. La carga máxima se fijó, en todos los ensayos, en $200 \mathrm{mN}$. Siguiendo el procedimiento de Oliver y Pharr $^{[1]}$ se determinaron el módulo de elasticidad, E, la dureza, $\mathrm{H}$ y la constante de ajuste de la curva P-h, B, de acuerdo a las siguientes expresiones:

$$
\begin{gathered}
E^{*}=\left[\frac{1-v^{2}}{E}+\frac{1-v_{\text {in }}^{2}}{E_{\text {in }}}\right]^{-1}=\frac{1}{c^{*} \sqrt{\mathrm{A}_{\text {max }}}}\left(\frac{d P}{d h}\right)_{\mathrm{P}_{\text {max }}} ; \\
H=\frac{P_{\text {max }}}{A} ; P=B h^{2}
\end{gathered}
$$

donde $c^{*}$ es una constante que para la punta Berkovich es 1,167 y A es el área proyectada de contacto.

\subsection{Recubrimientos NiCrBSi}

Por último, se realizaron ensayos de nanoindentación en dos recubrimientos de $\mathrm{NiCrBSi}$ con la siguiente composición del polvo proyectado $(\mathrm{Cr}$, $15,2 \%$; Fe, 3,6 \%; Si, 4 \%; B, 3,1 \%; C, 0,6 \% y Ni balance). Las técnicas de proyección térmica empleadas fueron plasma y fusión a la llama. En este segundo caso, los materiales fueron sometidos a un tratamiento térmico posterior. En la figura 3 se presenta un ejemplo de las curvas $\mathrm{P}-\mathrm{h}$ obtenidas en los ensayos de nanoindentación y los resultados de dureza y módulo de elasticidad.

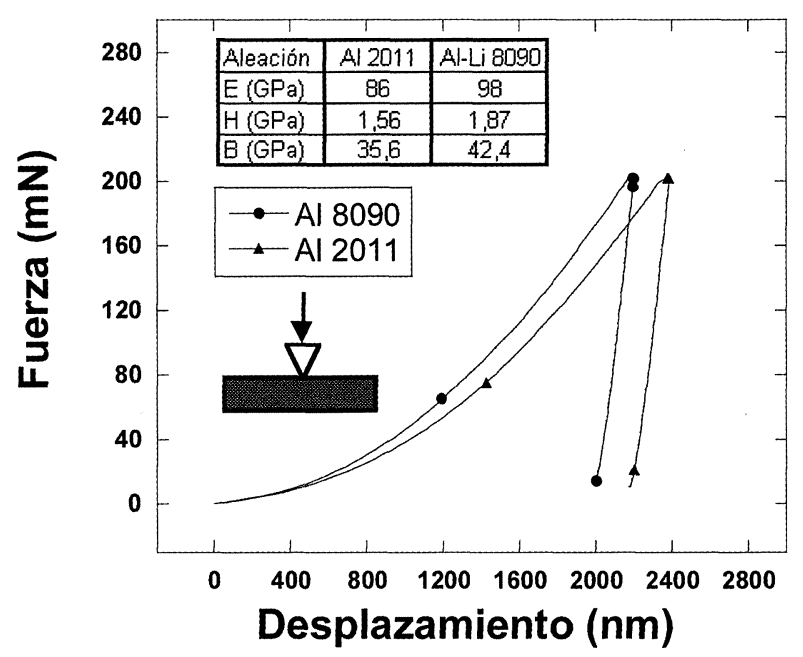

Figura 2. Curvas carga-desplazamiento de ensayos de nanoindentación para el aluminio.

Figure 2. Load-displacement curves from nanoindentation tests for aluminium alloys. 


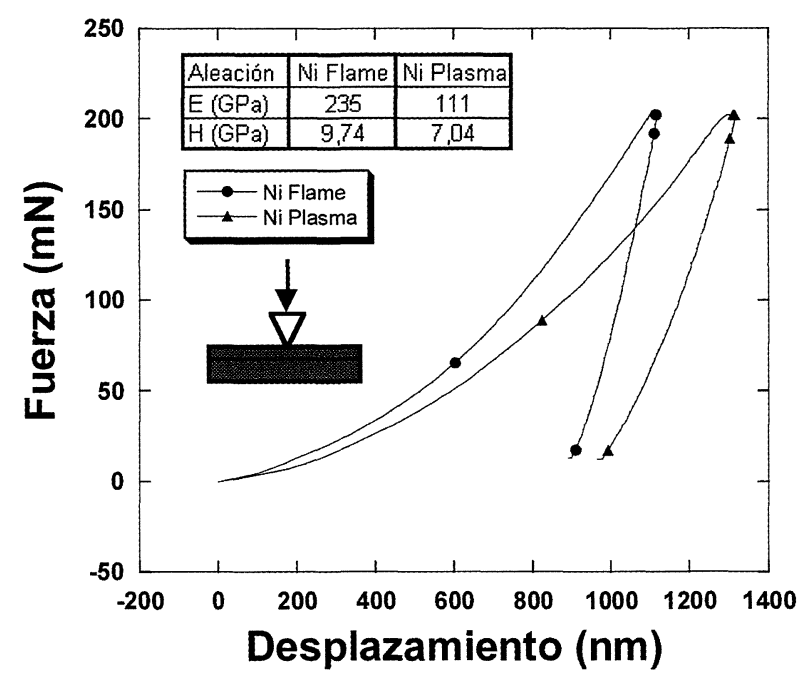

Figura 3. Curvas carga-desplazamiento de ensayos de nanoindentación para el $\mathrm{NiCrBSi}$.

Figure 3. Load-displacement curves from nanoindentation tests for NiCrBSi coating.

\section{ANÁLISIS Y DISCUSIÓN DE LOS RESULTADOS}

\subsection{Comparación entre tracción y nanoinden- tación para las aleaciones de aluminio}

Los datos obtenidos con ambos tipos de ensayo en las aleaciones de aluminio permiten realizar una discusión crítica de las posibilidades de las técnicas de nanoindentación. En primer lugar, ha de destacarse la diferencia apreciable obtenida en el módulo de elasticidad. Los ensayos de nanoindentación proporcionan valores de $\mathrm{E}$, alrededor de un $20 \%$ superiores a los obtenidos con el ensayo de tracción. Estas diferencias no pueden achacarse a la dispersión de los ensayos, pues se realizaron diez ensayos de nanoindentación en cada condición y material, siendo la desviación estándar mucho menor. Por otra parte los valores de $\mathrm{E}$ obtenidos en los ensayos de tracción son consistentes con el valor típico del módulo de elasticidad de las aleaciones de aluminio ensayadas.

La determinación del límite elástico y del índice de endurecimiento a partir de los ensayos de nanoindentación es una tarea mucho más compleja. La capacidad de endurecimiento del material tiene gran influencia en la distribución de la deformación plástica en el entorno del contacto probetaindentador. Al ensayar materiales con bajo índice de endurecimiento suele aparecer el fenómeno de apilamiento del material alrededor del indentador ( pile up), mientras que en materiales con alta capacidad de endurecimiento se observa experimental- mente un hundimiento (sink in). Estos fenómenos condicionan los resultados pues se modifica el área de contacto teórica.

Son varios los estudios que intentan incluir este efecto en el análisis. Uno de los más recientes, debido a Mata et al..$^{[5]}$, caracteriza el fenómeno a través del parámetro $\alpha$, definido como el cuadrado del cociente entre la distancia vertical desde el fondo de la huella al material situado en el límite de la zona de contacto y el máximo desplazamiento. La simulación del proceso utilizando elementos finitos proporciona una relación entre $\alpha$, el límite elástico y el índice de endurecimiento, $n$, para un material que responda a una ley potencial $\left(\sigma=K \varepsilon^{\mathrm{n}}\right)$. La constante $\alpha$ puede medirse directamente o determinarse a través de la dureza y la forma de curva carga-desplazamiento obtenida en el ensayo de nanoindentación. La aplicación de estos resultados a las aleaciones de aluminio ensayadas conducen a los resultados recogidos en la tabla I. Como puede observarse, el método es sensible a variaciones en el límite elástico y en el índice de endurecimiento, pero infravalora el primero y sobrevalora el segundo, en demasía.

En definitiva, aunque los resultados obtenidos con técnicas de nanoindentación están claramente relacionados con los parámetros característicos de la relación tensión-deformación del material, queda mucho trabajo por realizar para disponer de una metodología clara y general para determinar límite elástico e índice de endurecimiento.

\subsection{Recubrimientos NiCrBSi}

Los resultados obtenidos muestran que los materiales depositados por plasma son mucho menos rígidos y menos duros que los que fueron depositados por fusión a la llama y, posteriormente, sometidos a un tratamiento térmico. La microestructura característica de este tipo de materiales está condicionada por el proceso de proyección. Como puede apreciarse en la figura 4 , los recubrimientos

Tabla I. Comparación entre ensayos de tracción y resultados de Mata et al.

Table I. Tensile tests and results from Mata et al. comparison

\begin{tabular}{ccccc}
\hline & \multicolumn{2}{c}{ Al 8090} & \multicolumn{2}{c}{ Al 2011} \\
\hline & Mata et al. & Tracción & Mata et al. & Tracción \\
\hline $\begin{array}{c}\sigma_{0,2}(\mathrm{MPa}) \\
\mathbf{n}\end{array}$ & 361 & 508 & 235 & 325 \\
\hline
\end{tabular}

Rev. Metal. Madrid 40 (2004) 380-383 

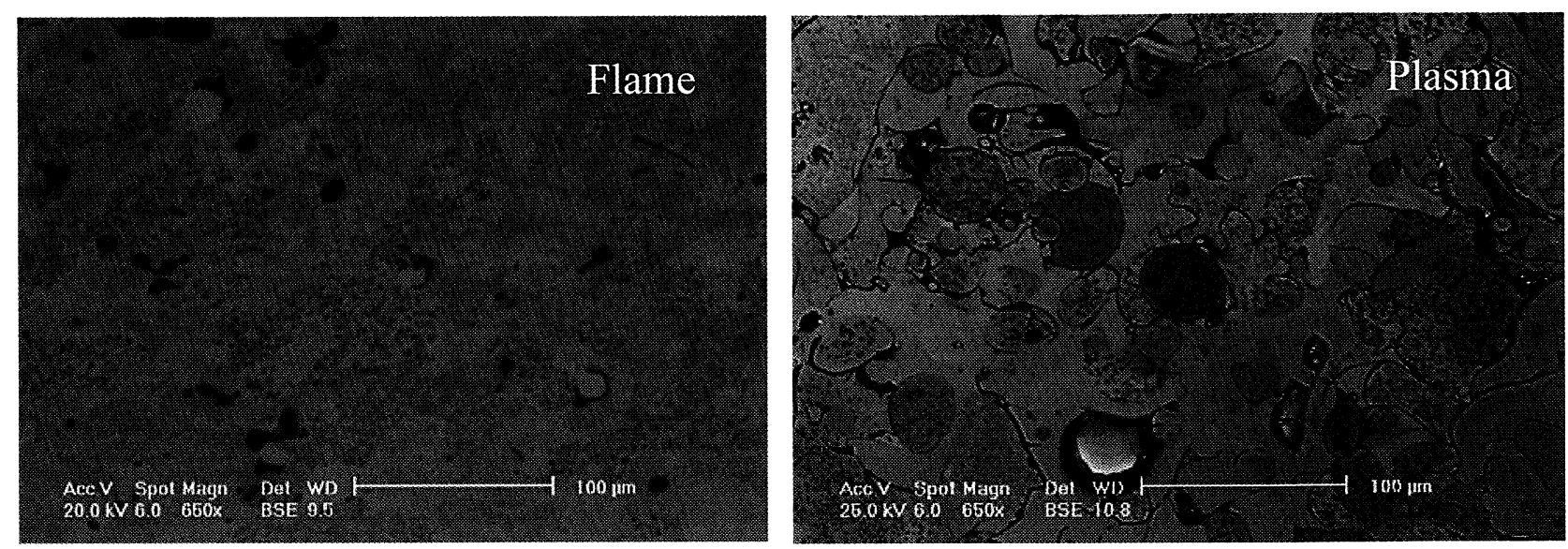

Figura 4. Micrografías de los recubrimientos $\mathrm{NiCrBSi}$.

Figure 4. Microstructure of the NiCrBSi coatings.

depositados por plasma presentan una gran cantidad de partículas separadas del resto del material por zonas oxidadas durante el proceso de proyección. Esto, debilita la cohesión interna del recubrimiento y conduce a curvas carga-desplazamiento claramente diferentes que proporcionan valores menores de módulo de elasticidad y dureza.

\section{CONCLUSIONES}

Los resultados de este trabajo permiten extraer las siguientes conclusiones:

- Los ensayos de nanoindentación son sensibles a variaciones de módulo elástico, límite elástico e índice de endurecimiento del material.

- Existen expresiones para determinar el módulo de elasticidad del material que proporcionan valores razonables aunque no iguales a los medidos en un ensayo de tracción.

- No se dispone de un método directo para obtener el límite elástico y el índice de endurecimiento del material. Los modelos de elementos finitos son un camino prometedor pero complejo y difícil de generalizar a una gran familia de materiales.
- A pesar de la difícil interpretación de los resultados, las técnicas de nanoindentación son un buen método de caracterización mecánica de recubrimientos.

\section{Agradecimientos}

Los autores desean agradecer al Ministerio de Ciencia y Tecnología el apoyo económico recibido para la realización de esta investigación a través del proyecto MAT2001-3528-C03-03.

\section{REFERENCIAS}

[1] W.C. Oliver y G.M. Pharr, J. Mater. Res. 7 (1992) 15641583

[2] A.C. FISHER-CRIPPS, Vacuum 58 (2000) 569-585.

[3] J. Maltzbender, J.M.J. Den Toonder, A.R. Balkenende y G. DE With, Mater. Sci. Eng. R 36 (2002) 47-103.

[4] A.E. Giannakopoulos y S. Suresh, Scri. Materialia 40 (1999) 1191-1198.

[5] M. Mata, M. Anglada y J. Alcala, J. Mater. Res. 17 (2002) 964-976.

[6] V. Higuera, F.J. Belzunce, A. Carriles y S. Poveda, Tribol. Int. 34 (2001) 161-169. 